\title{
InAs quantum dots embedded into anti-modulation-doped GaAs superlattice structures
}

\author{
W.T. Masselink, H. Kissel, U.Mueller, C. Walther \\ Humboldt-Universitat zu Berlin, Dept. of Physics, Invalidenstrasse 110, D-10115 Berlin, Germany
}

Yu.I. Mazur, G.G .Tarasov, M.P. Lisitsa, S.R. Lavoric, Z.Ya. Zhuchenko

Institute of Semiconductor Physics, National Academy of Sciences, Prospect Nauki 45, 03650 Kiev, Ukraine

\begin{abstract}
Photoluminescence (PL) spectra of anti-modulation-doped GaAs superlattice structures containing thin InAs films of about 1-2.5 monolayers grown on semi-insulating (001)oriented GaAs substrates at different temperatures are studied. The size distribution of InAs quantum dots (QD's) is found to be bimodal at the higher substrate growth temperature $\left(T_{G}=505{ }^{\circ} \mathrm{C}\right)$ and is transformed into multimodal for the decreased growth temperature $\left(T_{G}=420{ }^{\circ} \mathrm{C}\right)$ and growth interruption applied. For the first time we demonstrate the strong coupling between modes, which stabilizes the PL magnitude and the full width at half maximum of large index QD modes within a certain temperature interval $(50-150 \mathrm{~K})$ due to feeding of the radiative transitions from non-radiative decay and carrier transfer arising from decaying excitonic states of the low index QD mode.
\end{abstract}

Keywords: quantum dots, InAs, GaAs, superlattice, photoluminescence

Paper received 02.09.99; revised manuscript received 11.11.99; accepted for publication 14.03.00.

\section{Introduction}

A morphological transition from two-dimensional (2D) to three-dimensional (3D) growth of highly strained InAs layers on GaAs substrates by molecular beam epitaxy, known as the Stranski-Krastanov growth mode, results in three-dimensional islands (quantum dots) appearance and occurs when the InAs layer thickness exceeds the critical value (1.7 monolayers, ML) [1], being recognized that at the early stage of transition the dots do not possess a definite crystallographic shape. The existence of critical thickness is predicted theoretically due to growth kinetics analysis at thermodynamic equilibrium. The well developed dots are observed at InAs coverage of about $1.2 \mathrm{~nm}$ with a remarkably homogeneous size distribution, stable under prolonged growth interruption. Nevertheless, recent studies have shown that the change of InAs morphology strongly depends on kinetics factors and can be characterized by smooth $2 \mathrm{D}-3 \mathrm{D}$ transition in the region of InAs coverages far below the predicted critical value [2-5]. It was established in part that the different growth conditions may result either in fabrication of highquality 2D layers with subsequent QD nucleation, or in the 3D growth with an earlier appearance of QD's.
Strong influence of the growth conditions on the size, shape, and areal density of QD's was demonstrated recently by PL study, and atomic force microscopy (AFM) study permitting to trace the initial dot formation with evolution of the wetting layer (WL) and precursor states into genuine quantum well (QW) [6-8]. It has been shown that by changing the substrate temperature and arsenic pressure it is possible to change the size, density, and quantum efficiency of QD's, which are influenced by not only the amount of InAs deposited but also by the diffusion length of the adatoms. The emission bands with a full width at a half of the maximum (FWHM) of order of 20-30 meV were typically observed in self-assembled QD's for growth conditions favoring the nucleation of a particular pattern of quantized families of dots with a remarkable size uniformity [9]. Inasmuch, the multimodes growth regime is declared. In present communication we report about, at least, bimodal behavior of the PL spectra from InAs QW embedded in the superlattice, which is found to be strongly modified by growth temperature variation with complementary growth interruption (GI) applied. Such procedure allows to share the InAs material to the QD's of smaller size and consequently to stabilize them. 


\section{Experimental details}

The samples investigated in this study are anti-modulation-doped GaAs superlattice structures containing thin layers of InAs grown on semi-insulating (001)-oriented GaAs substrates in a Riber 32-P gas-source molecularbeam epitaxy (GSMBE) system with growth rates of 2.5, 3.125 , and $0.2 \AA /$ s for GaAs, $\mathrm{AlGaAs}$, and InAs, respectively. All samples consist of 10 periods of $60 \AA$ $\mathrm{Al}_{0.2} \mathrm{Ga}_{0.8} \mathrm{As}, 30 \AA \mathrm{GaAs}, 60 \AA \mathrm{GaAs}: \mathrm{Si}\left(N_{D}=2 \cdot 10^{18}\right.$ $\mathrm{cm}^{-3}$ ), $60 \AA$ GaAs, $4 \AA$ to $8 \AA$ InAs, $60 \AA$ GaAs, $60 \AA$ GaAs:Si, $30 \AA \mathrm{GaAs}, 60 \AA \mathrm{Al}_{0.2} \mathrm{Ga}_{0.8} \mathrm{As}$, subsequently capped with a $50 \AA$ GaAs:Si layer. There are two sets of samples: those with a relatively high substrate temperature $T_{G}$ during the InAs growth $\left(T_{G}=505^{\circ} \mathrm{C}\right)$ and those with a relatively low $T_{G}\left(T_{G}=420^{\circ} \mathrm{C}\right)$. The growth temperature of all structures was $530{ }^{\circ} \mathrm{C}$, except of the InAs and the adjacent GaAs layers which were grown at $T_{G}$ after a short GI allowing the substrate to reach this lower temperature. Double crystal X-ray diffraction together with a simulation of the double X-ray rocking curve were used to verify the completed structures. The average value for the coverage of InAs layers is obtained by the 2D simulation also in the presence of QD's. We found a linear dependence of the nominal InAs coverage $d_{\text {InAs }}$, on the InAs growth time (excluding GI during the dot formation) [10].

Reference samples with a single InAs layer were studied in a $200 \mathrm{MV}$ Hitachi H8110 transmission electron microscope (TEM) for the characterization of shape, size, and density of the InAs QD's. Plane-view specimens were prepared by backside mechanical polishing and chemical dissolution of the GaAs substrate. Our samples grown near the high (low) temperature limit for the self-formation of InAs QD's in GaAs [11] contain dots with different lateral sizes and areal density $\left(D_{a}\right)$ depending on InAs coverage $(L)$. So, for $T_{G}=505^{\circ} \mathrm{C}$ and $L \sim 3 \mathrm{ML}$ the QD's are with an average base length $b=14.5 \mathrm{~nm}$ and $D_{a}=3.5 \cdot 10^{10} \mathrm{~cm}^{-2}$, whereas at $T_{G}=420{ }^{\circ} \mathrm{C}$ and $L \sim 2 \mathrm{ML}$ these parameters were of $b=8 \mathrm{~nm}$ and $D_{a}=1.7 \cdot 10^{11} \mathrm{~cm}^{-2}$, respectively. Under diminishing the $L$ value at the same temperature, the appearance of QD's of smaller $b$ is observed.

The PL was excited by the $514.5 \mathrm{~nm}$ line of a cw Ar ${ }^{+}$ laser and the excitation densities were in the range of $1 \mathrm{~mW} / \mathrm{cm}^{2}$ to $20 \mathrm{~W} / \mathrm{cm}^{2}$. The PL radiation was dispersed through a 3/4-m Czerny-Turner scanning spectrometer, with a spectral resolution better than $0.1 \mathrm{meV}$, and detected using a lock-in amplifier with a thermoelectrically cooled photomultiplier tube (containing a $\mathrm{GaAs}(\mathrm{Cs})$ photocathode) or a $\mathrm{LN}_{2}$ cooled high-purity Ge detector. The samples were mounted in an Oxford Spectromag 4000 system allowing measurements in magnetic fields up to 7 $\mathrm{T}$ and at temperatures from 1.7 to $300 \mathrm{~K}$.

\section{Results and discussion}

Representative low-temperature (6 K) PL spectra of InAs QD's are shown in Fig. 1. It demonstrates the spectra

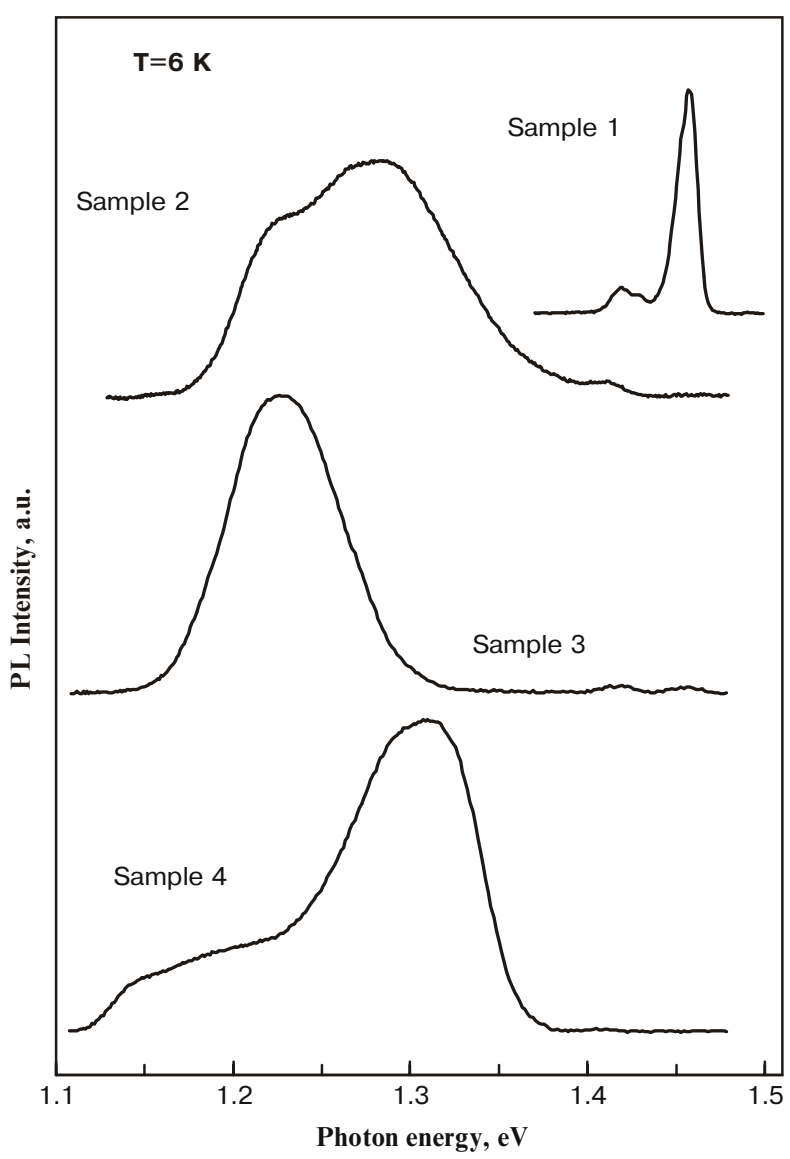

Fig. 1. PL spectra from InAs quantum dots at different InAs coverage $(L)$ and at different growth conditions $\left(T_{G}\right)$ : a) sample $1-L=1.33 \mathrm{ML}, T_{G}=505{ }^{\circ} \mathrm{C}$; b) sample $2-L=1.93 \mathrm{ML}, T_{G}=505$ ${ }^{\circ} \mathrm{C}$; c) sample $3-L=2.5 \mathrm{ML}, T_{G}=505{ }^{\circ} \mathrm{C}$; d) sample $4-L=2.66$ $\mathrm{ML}, T_{G}=420{ }^{\circ} \mathrm{C}$.

evolution for different InAs coverage $L$ in the samples grown with a substrate temperature of about $505^{\circ} \mathrm{C}$. In the case of $L=1.33 \mathrm{ML}$ (Sample 1) a WL layer peak is observed at about $1.45 \mathrm{eV}$, accompanied by a red-side satellite at a position of $1.42 \mathrm{eV}$. The peak at $1.45 \mathrm{eV}$ is related to recombination of the heavy-hole free exciton in a $2 \mathrm{D}$ InAs layer. The WL thickness coincides with the nominal InAs one. As the InAs coverage is increased, the WL peak exhibits a red shift. The nature of the low-energy peak has been widely discussed $[2,12,13]$ and is ascribed to either self-aggregated QD's (SAQD's) at the interface between InAs and GaAs layer or to a carrier localization by the potential fluctuation with account for the SAQD's with a continuous distribution of radii at the interface of the quantum well. The two-hump broad PL feature (FWHM $\sim 120 \mathrm{meV}$ ), shown in Fig. 1, obviously demonstrates a developed QW assemblage structure in sample 2 with $L=1.93 \mathrm{ML}$. Further increase of the InAs coverage leads to a narrowing the PL FWHM (FWHM $\sim 80 \mathrm{meV}$ ) and shifts the PL peak towards lower energies (Fig. 1, sample 3). The distribution in the lateral sizes of 


\section{W.T. Masselink et al.: InAs quantum dots embedded into anti-modulation-doped GaAs...}

QD's results mainly in the peak width at low temperature and low excitation intensity. Therefore, as further InAs is deposited, the smaller dots turn into regular sized ones of a larger diameter. The total red-shift of the PL peak reflects here a smaller confinement in a large-scaled QD. For lower substrate temperature, the diffusion length of the adatoms decreases and the smaller sized QD formation is expected. The QD density is also dependent on the substrate temperature, because the density of the nucleation centers is sensitively dependent on the surface diffusion of the adatoms [8]. The usage of growth interruption results normally in the formation of larger sized QD's of regular size distribution [1]. Combining GI with the variation of the substrate temperature it is possible to affect strongly the QW size distribution with a formation of a preferable size mode. Fig. 1 shows the PL spectrum obtained from the sample 4, grown with a substrate temperature of $T_{G}=420^{\circ} \mathrm{C}$ and GI. An enhanced PL intensity is observed at $1.30 \mathrm{eV}$ for InAs coverage of $2.33 \mathrm{ML}$. It reflects the enlarged quantity of smaller QD's with simultaneous formation of regular modes of larger sizes. The spectra obtained have been studied under various conditions: temperature range from $T=6 \mathrm{~K}$ up to room temperature, excitation density varied from $0.01 \mathrm{~W} / \mathrm{cm}^{2}$ up to $20 \mathrm{~W} / \mathrm{cm}^{2}$, and magnetic field $(B)$ changed from $B=0 \mathrm{~T}$ up to $7 \mathrm{~T}$. The study in magnetic field shows no visible effects both in the PL peak line shape and in the PL peak position, as it was expected for the small-sized QD's ( $10 \mathrm{~nm}$ ) and broad PL feature. The study under excitation intensity variation shows a remarkable reproducibility of the PL shape, excluding in such way the contribution of the spectral components which stem normally from excited states of the QD's. The PL intensity depends linearly on the excitation density over three orders of magnitude, i.e. from the moderate level of $20 \mathrm{~W} / \mathrm{cm}^{2}$ down to $0.01 \mathrm{~W} / \mathrm{cm}^{2}$.

A dramatic change in the PL line shape occurs for temperature variation. Fig. 2 depicts this behavior for samples 2 (a) and 4 (b). It is clearly seen that the highenergy component decays rapidly with the temperature elevation, whereas the low-energy one preserves its shape up to temperatures of about $200 \mathrm{~K}$. In order to make a more precise assignment, the PL spectrum of Sample 2 is deconvoluted into two Gaussians. Fig. 3 shows the temperature dependence of both band characteristics: energies (Fig. 3a), FWHM (Fig. 3b), and peak magnitudes
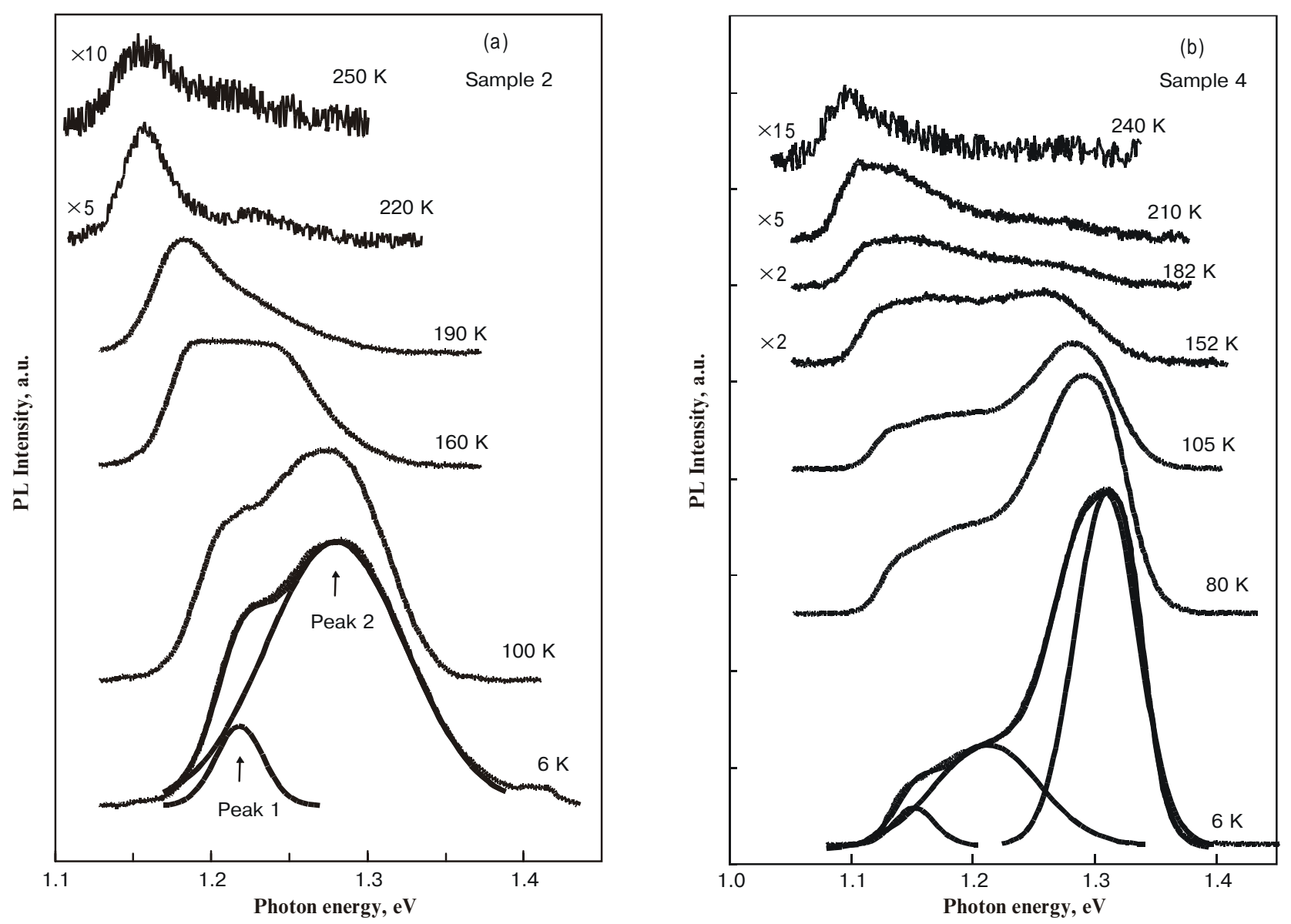

Fig. 2. Temperature dependence of PL spectra for samples 2 (a) and 4 (b). Size modes assignment is performed due the PL shape line deconvolution into Gaussians - dashed lines. 
W.T. Masselink et al.: InAs quantum dots embedded into anti-modulation-doped GaAs...

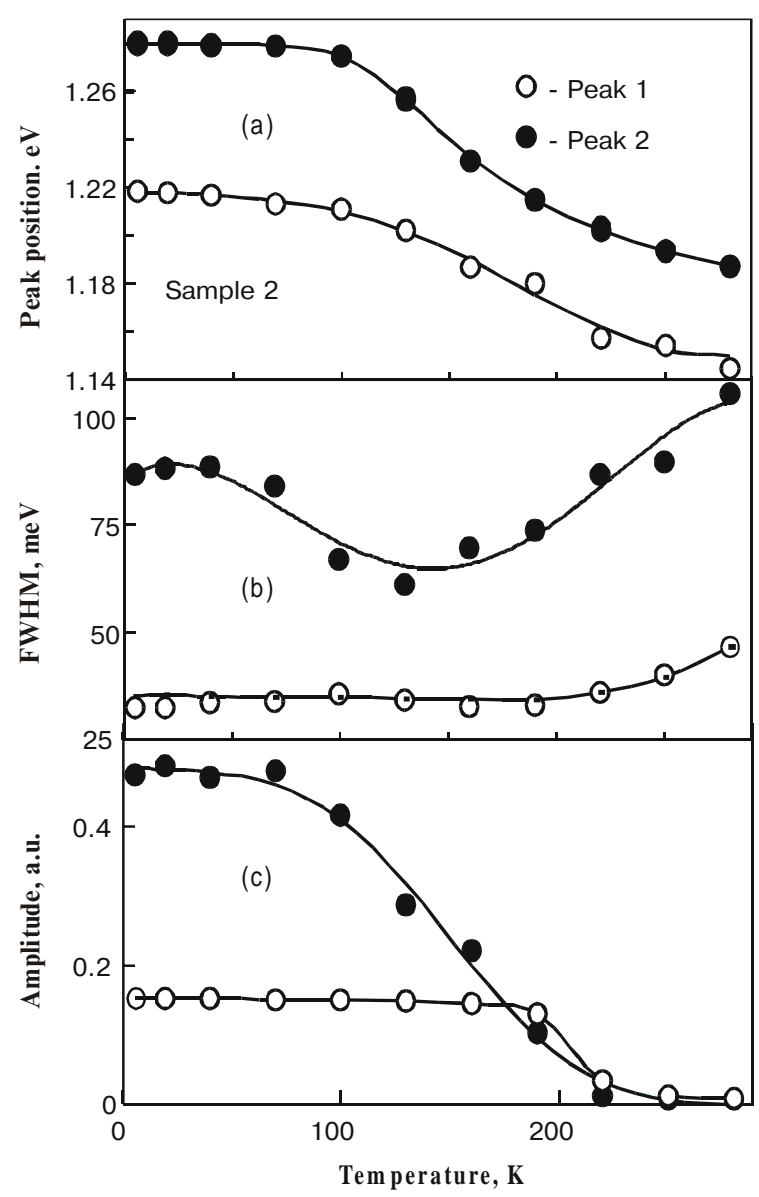

Fig. 3. Temperature dependence of band parameters (sample 2) for deconvoluted PL components: a) energy positions; b) FWHM; c) peak magnitudes.

(Fig. 3c). The behavior observed gives strong evidence of the bimodal distribution of the QD sizes: the mode 2 is responsible for peak 1 at $1.22 \mathrm{eV}$ and assembled from a larger sized QD, and the mode 1 is aggregated from a smaller sized QD and gives rise to the peak at $1.28 \mathrm{eV}$. The energy position of each of the PL components follows the Varshni law with the InAs parameters [14,15]. However, the behavior of the FWHM and the peak magnitude (integral intensity) seems to be more indicative in the case. One can clearly see that the amplitude of the low-energy peak is invariable up to a temperature of $200 \mathrm{~K}$, whereas the high-energy peak strongly decreases with temperature elevation. The behavior of FWHM correlates with that of peak magnitude. Similar trends are observed for integral intensities of both peaks, too. Peak 1 temperature dependence is determined by the excitonic mechanism of QD recombination. The observation of high luminescence efficiency from QD's of smaller sizes (low index mode) indicates an additional confinement of the InAs excitons in lateral directions. The high luminescence efficiency implies also that the phonon bottleneck effect is not actual for the case. Up to a temperature of $\sim 60 \mathrm{~K}$ the peak position is almost invariable due to the exciton lo- calization in a certain potential minimum. When the thermal energy becomes comparable to the exciton binding energy, free carriers arising due to the exciton dissociation can escape from the QD's in part due to tunneling between the nearby QD's. The thermalized carrier can relax over a long distance and find a lower local-energy minimum, resulting in a shrinkage of the linewidth. Besides, the tunneling occurence will be more frequent for the QD's with a predominane size (within their Gaussian distribution), and this also can contribute to the reduction of FWHM observed for the peak 1. With further temperature increase above $150 \mathrm{~K}$, the electron-phonon scattering and thermal distribution are of importance, giving rise to the FWHM enlargement. From the Arrhenius plot for the integrated intensity, the thermal activation energy is extracted. We used the high-energy part of the plot, when the thermal quenching of the PL is mainly related to the escape process of the dissociated exciton into the corresponding barriers. For peak 1, it is found to be of 135 $\mathrm{meV}$, being comparable with the energy difference between the PL emission peak in InAs QD's and that in the WL. In sample 2, the WL emission energy is directly measured and occurs to be $1.42 \mathrm{eV}$. In fact, the variation of the WL energy is strongly dependent on growth conditions. The behavior of peak 2 reflects the coexistence of excitonic recombination with carrier transfer between QD's with different energy levels and QD's of different modes, respectively. Indeed, the exciton created by light absorption in 2D-InAs region and in GaAs barriers are effectively captured into the QD's, where they recombine. Both carrier tunneling between InAs QD's and carrier percolation through the 2D-InAs layer may contribute to the carrier transfer. The efficiency of these processes has to be high in assemblage with small average distance between QD's. Moreover, the 2D-InAs layer connects the QD's of different sizes and, consequently, of different emission energies. Taking into account the excitonic nature of the PL, the excitonic thermal activation, and carrier transfer, including the tunnel transfer among or from an individual $\mathrm{QW}$, we are able to explain the peak 2 behavior. Indeed, the relaxation of carriers and their radiative recombination effected by temperature occur for both separate size modes (assemblages). However, the nonradiative decay of the PL for the mode 1 serves as a complementary channel of feeding for QD's of mode 2. Being of a smaller density, these latter QD's are saturated due to the carrier flux from the decaying radiative states belonging to the mode 1 . This saturation is clearly manifested in temperature behavior of peak 2. Both its magnitude and FWHM stay invariable at the temperature elevation in contrast to strong quenching of the PL signal from QD's of mode 1. The compensation is governed by the rate equation. At a temperature as high as $200 \mathrm{~K}$ the carrier flux from the decaying QD states of mode 1 is exhausted and can not completely compensate the PL signal decrease in the mode 2 . The peak 2 magnitude decreases, accompanied by the corresponding change of the FWHM. To the best of our knowledge, such a clear demonstration of the bimodal behavior of PL with tempera- 


\section{W.T. Masselink et al.: InAs quantum dots embedded into anti-modulation-doped GaAs...}

ture-induced carrier transfer between the size modes of QD's is presented for the first time.

A similar temperature behavior is observed for the sample 4 (Fig. 2b). The broad low-energy shoulder is deconvoluted into two comparatively narrow peaks positioned at $1.15 \mathrm{eV}$ and $1.22 \mathrm{eV}$, respectively. This latter energy coincides very close with that for the peak 2 of sample 2, giving hint on the regular structure of this size mode. The high energy peak occurs at $1.32 \mathrm{eV}$, being indicative for the mode of smaller sizes (low index mode). The spectral deconvolution supports the co-existence of three modes simultaneously. The temperature elevation favors again the subsequent decay of high energy peak, then the peak at $1.22 \mathrm{eV}$, and results finally in the peak at $1.10 \mathrm{eV}$ for the temperature reaching $T=250 \mathrm{~K}$. This corresponds to well-developed large sized QD's, observed under GI applied [1]. Principally, the temperature behavior of the PL components representing different size mode, is similar to that observed for the sample 2. While the high-energy component quenches rapidly, the two lowenergy ones preserve their magnitudes and FWHM. Then at $T=150 \mathrm{~K}$ the peak at $1.22 \mathrm{eV}$ starts to quench. It is worth to note that the quenching of the small sized QD's is determined by carrier transfer, including the tunneling. This latter strongly depends on the average distance between the QD's. Inasmuch the distance is smaller in the sample 4, i.e. with respect to higher QD density, the temperature-induced decay of the PL signal has to be of much higher rate. Indeed, we observe the high energy peak in sample 4 decaying about a factor of 5 stronger than that in sample 2 within the temperature interval $50-150 \mathrm{~K}$.

\section{Summary}

In summary, we studied the well-pronounced distribution of QD sizes in different modes observed for increasing InAs coverage. This size distribution is bimodal at the higher substrate growth temperature $\left(T_{G}=500{ }^{\circ} \mathrm{C}\right)$ and transformed into a multimodal one for decreased growth temperature $\left(T_{G}=420^{\circ} \mathrm{C}\right)$ and applied GI. For the first time, we demonstrate the strong coupling between modes stabilizing PL magnitude and FWHM of larger index QD modes within a certain temperature interval (50-150 K) due to feeding of the radiative transitions by non-radiative decay and carrier transfer from decaying excitonic states of low index QD mode.

\section{Acknowledgments}

This work is supported by NATO linkage grant.

\section{References}

1. M. Grundmann, Pseudomorphic InAs/GaAs quantum dots on low index planes, in Advances in Solid State Physics 35 , Ed. R. Helbig, pp. 123-154, Vieweg, Braunschweig (1996).

2. A. Polimeni, A. Patane, M. Capizzi, F. Martelli, L. Nasi, and G. Salviati, Self-aggregation of quantum dots for very thin InAs layers grown on GaAs // Phys. Rev. B 53(8), pp. R4213-R4216 (1996)

3. T. R. Ramachandran, R. Heitz, P. Chen, and A. Madhukar, Mass transfer in Stranski-Krastanow growth of InAs on GaAs // Appl. Phys. Lett. 70(5), pp. 640-642 (1997).

4. R. Heitz, T. R. Ramachandran, A. Kalburge, Q. Xie, I. Mukhametzanov, P. Chen, and A. Madhukar, Observation of reentrant $2 \mathrm{D}$ to $3 \mathrm{D}$ morphology transition in highly strained epitaxy: InAs on GaAs // Phys. Rev. Lett. 78(21), pp. 40714074 (1997).

5. A. Patane, M. G. Alessi, F. Intonti, A. Polimeni, M. Capizzi, F. Martelli, L. Nasi, L. Lazzarini, G. Salviati, A. Bosacchi, and S. Franchi, Self-aggregated InAs quantum dots in GaAs // J. Appl. Phys. 83(10), pp. 5529-5535 (1998).

6. I. Kamiya, I. Tanaka, and H. Sakaki, Optical properties of near surface-InAs quantum dots and their formation processes // Physica E 2, pp. 637-642 (1998).

7. S. P. Guo, A. Shen, Y. Ohno, and H. Ohno, InAs quantum dots and dashes grown on (100), (211)B, and (311)B GaAs substrates // Physica E 2, pp. 672-677 (1998).

8. L. Chu, M. Arzberger, G. Bohm, and G. Abstreiter, Influence off growth conditions on the photoluminescence of selfassembled InAs/GaAs quantum dots // J. Appl. Phys. 85(4), pp. 2355-2362 (1999).

9. M. Colocci, F. Bogani, L. Carraresi, R. Mattolini, A. Bossacchi, S. Franchi, P. Frigeri, S. Taddei, and M. RosaClot, Size quantization patterns in self-assembled InAs/GaAs quantum dots // Superlattices and Microstructures 22(1), pp. 81-84 (1997).

10. C. Walther, B. Herrmann, I. Hahnert, W. Neumann, and W. T. Masselink, Electrical transport in superlattices containing InAs quantum dots in GaAs and InP // Superlattices and Microstructures 25(1/2), pp. 53-56 (1999).

11. Q. Xie, N. P. Kobayashi, T. R. Ramachandran, A. Kalburge, P. Chen, and A. Madhukar, Strained coherent InAs quantum box islands on GaAs (100): Size equalization, vertical self-organization, and optical properties // J. Vac. Sci. Technol. B 14(3), pp. 2203-2207 (1996).

12. R. Cingolani, O. Brandt, L. Tapfer, G. Scamarcio, G. C. La Rocca, and K. Ploog, Exciton localization in submonolayer InAs/GaAs multiple quantum wells // Phys. Rev. B 42(5), pp. 3209-3912 (1990); O. Brandt, R. Cingolani, H. Lage, G Scamarcio, L. Tapfer, and K. Ploog, Radiative decay of excitonic states in bulklike GaAs with a periodic array of InAs lattice planes // Phys. Rev. B 42(17), pp. 11396-11399 (1990).

13. A. Polimeni, D. Marangio, M. Capizzi, A. Frova, and F. Martelli, Giant photoluminescence enhancement in deuterated highly strained InAs/GaAs quantum wells // Appl. Phys. Lett. 65 (10), pp. 1254-1256 (1994).

14. Y. P. Varshni, Temperature dependence of the energy gap in semiconductors // Physica 34(1), pp. 149-154 (1967).

15. L. Brusaferri, S. Sanguinetti, E. Grilli, M. Guzzi, A. Bignazzi, F. Bogani, L. Carraresi, M Colocci, A. Bosacchi, P. Frigeri, and S. Franchi, Thermally activated carrier transfer and luminescence line shape in self-organized InAs quantum dots // Appl. Phys. Lett. 69(22), pp. 3354-3356 (1996). 